\title{
Change Ideas for First Quality Improvement Project
}

\author{
Deepak Chawla ${ }^{1}$ (1)
}

Received: 25 January 2020 / Accepted: 30 January 2020 / Published online: 8 February 2020

(C) Dr. K C Chaudhuri Foundation 2020

Using evidence-based interventions while treating sick or preterm neonates is the cornerstone of high-quality clinical care. Nasal continuous positive airway pressure (NCPAP) is the modality of choice for respiratory support during initial management of respiratory distress in neonates. However, nasal trauma associated with the use of various nasal interfaces is a common complication. Proper fixation of the interface, use of a physical barrier between skin and interface, and good nursing care can reduce the incidence of nasal trauma. This is easier said than done and a system-based implementation of nasal trauma prevention strategy is needed. Mariam and Buddhavarapu in their study, published in this issue of the journal, successfully address this challenge with about $90 \%$ reduction in the prevalence of nasal injury [1]. The quality improvement strategy used by the authors are based on the change ideas which can be applied to any healthcare setting, especially those in the resource-constrained environment or those looking for low-hanging fruit at the beginning of improvement journey.

Standardization: One common reason of suboptimal outcomes is the variability in care processes observed within a health facility. This may result from disagreement about clinical protocols amongst different members of the clinical care team or simply lack of any agreed upon protocols. This leads to excessive common cause variation (too much variation not ascribed to any special event). Use of checklists and pre-packed kits as used in this study are two methods to reduce variability and standardize the care process. This type of standardization can be used to rapidly improve many care practices in a nascent unit. Examples include standard

Deepak Chawla

drdeepakchawla@gmail.com

1 Department of Neonatology, Government Medical College Hospital, Chandigarh 160 030, India operating procedures for common areas of disagreement like use of antibiotics and introduction of enteral feeds. Reducing common cause variation and bringing the process under control of a standard operating procedure can be the first step in improving any care process.

Data review: Cognitive errors like availability bias, aggregate bias, anchoring, base rate neglect and confirmation bias can cloud clinical diagnosis and therefore the judgement about incidence of events [2]. As a result, one may refute the very idea of the need of quality improvement in his/her unit. One way to reduce some of the cognitive errors is the collection and regular review of data about the events of interest. For example, collecting data about incidence of hypothermia at admission to a unit, rate of exclusive breastfeeding, and incidence and severity of NCPAPrelated injury itself may make the healthcare professionals of the unit aware about the burden of problem. This awareness itself is the beginning of improvement.

Operating rules: The Donabedian model describes three elements of a healthcare system: structure, process and outcome [3]. The point of care quality improvement (POCQI) model focusses on improving the process of healthcare using the given structure to improve the patient outcomes. However, system reengineering or redesigning a process may not succeed unless operating rules are aligned with the improvement efforts. Operating rules consist of values, customs, and habits; in other words, the culture of improvement [4]. Having a sense of urgency to improve, understanding the importance of teamwork (vis-à-vis individual heroism) and ingraining the habit of improvement in everyone are essentials without which quality will not improve or revert back to square once priorities shift elsewhere.

Neonatal clinical units are complex care systems with interactions of many processes, departments and personnel. Bigger the unit, more daunting it may feel to start a quality improvement project. Regular data review and standardization of care processes can be the starting point. 


\section{Compliance with Ethical Standards}

Conflict of Interest None.

\section{References}

1. Mariam S, Buddhavarapu S. Impact of systematic training and CPAP checklist in the prevention of NCPAP related nasal injuries in neonates- a quality improvement study. Indian J Pediatr [Internet]. 2020. https://doi.org/10.1007/s12098-019-03146-5.
2. Croskerry P. The importance of cognitive errors in diagnosis and strategies to minimize them. Acad Med. 2003;78:775-80.

3. Donabedian A. The quality of care. How can it be assessed? JAMA. 1988;260:1743-8.

4. Langley GJ. The improvement guide: a practical approach to enhancing organizational performance, 2nd ed. San Francisco: Jossey-Bass; 2009.

Publisher's Note Springer Nature remains neutral with regard to jurisdictional claims in published maps and institutional affiliations. 